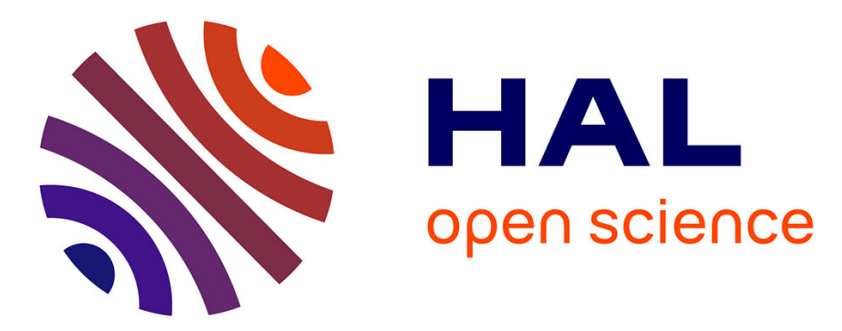

\title{
Spin orbitronics at a topological insulator-semiconductor interface
}

T. Guillet, C. Zucchetti, A. Marchionni, A. Hallal, P. Biagioni, C. Vergnaud, A. Marty, H. Okuno, Aurélien Masseboeuf, M. Finazzi, et al.

\section{- To cite this version:}

T. Guillet, C. Zucchetti, A. Marchionni, A. Hallal, P. Biagioni, et al.. Spin orbitronics at a topological insulator-semiconductor interface. Physical Review B, 2020, 101 (18), 10.1103/PhysRevB.101.184406 . hal-02991430

\section{HAL Id: hal-02991430 \\ https://hal.science/hal-02991430}

Submitted on 16 Nov 2020

HAL is a multi-disciplinary open access archive for the deposit and dissemination of scientific research documents, whether they are published or not. The documents may come from teaching and research institutions in France or abroad, or from public or private research centers.
L'archive ouverte pluridisciplinaire HAL, est destinée au dépôt et à la diffusion de documents scientifiques de niveau recherche, publiés ou non, émanant des établissements d'enseignement et de recherche français ou étrangers, des laboratoires publics ou privés. 


\title{
Spin orbitronics at a topological insulator-semiconductor interface
}

\author{
T. Guillet, ${ }^{1, *}$ C. Zucchetti, ${ }^{2, *}$ A. Marchionni $\odot,{ }^{2}$ A. Hallal $\odot,{ }^{1}$ P. Biagioni, ${ }^{2}$ C. Vergnaud, ${ }^{1}$ A. Marty, ${ }^{1}$ H. Okuno, ${ }^{3}$ \\ A. Masseboeuf, ${ }^{1}$ M. Finazzi ${ }^{\circ},{ }^{2}$ F. Ciccacci, ${ }^{2}$ M. Chshiev, ${ }^{1}$ F. Bottegoni $\odot,{ }^{2}$ and M. Jamet ${ }^{1}$ \\ ${ }^{1}$ Université Grenoble Alpes, CEA, CNRS, Grenoble INP, IRIG-Spintec, 38000 Grenoble, France \\ ${ }^{2}$ LNESS-Dipartimento di Fisica, Politecnico di Milano, Piazza Leonardo da Vinci 32, 20133 Milano, Italy \\ ${ }^{3}$ Université Grenoble Alpes, CEA, IRIG-MEM, 38000 Grenoble, France
}

(Received 24 November 2019; accepted 20 April 2020; published 5 May 2020)

\begin{abstract}
Topological insulators (TIs) hold great promise for new spin-related phenomena and applications thanks to the spin texture of their surface states. However, a versatile platform allowing for the exploitation of these assets is still lacking due to the difficult integration of these materials with the mainstream Si-based technology. Here, we exploit germanium as a substrate for the growth of $\mathrm{Bi}_{2} \mathrm{Se}_{3}$, a prototypical TI. We probe the spin properties of the $\mathrm{Bi}_{2} \mathrm{Se}_{3} / \mathrm{Ge}$ pristine interface by investigating the spin-to-charge conversion taking place in the interface states by means of a nonlocal detection method. The spin population is generated by optical orientation in $\mathrm{Ge}$ and diffuses toward the $\mathrm{Bi}_{2} \mathrm{Se}_{3}$, which acts as a spin detector. We compare the spin-to-charge conversion in $\mathrm{Bi}_{2} \mathrm{Se}_{3} / \mathrm{Ge}$ with the one taking place in Pt in the same experimental conditions. Notably, the sign of the spin-to-charge conversion given by the TI detector is reversed compared to the $\mathrm{Pt}$ one, while the efficiency is comparable. By exploiting first-principles calculations, we ascribe the sign reversal to the hybridization of the topological surface states of $\mathrm{Bi}_{2} \mathrm{Se}_{3}$ with the $\mathrm{Ge}$ bands. These results pave the way for the implementation of highly efficient spin detection in TI-based architectures compatible with semiconductor-based platforms.
\end{abstract}

DOI: 10.1103/PhysRevB.101.184406

\section{INTRODUCTION}

In the past decade, the discovery of topological insulators (TIs) has promised a breakthrough in the efficiency of spincharge interconversion phenomena. Indeed, TIs are known to host topologically protected surface states (TSSs) leading to spin-momentum locking [1]. This has been experimentally verified by means of photoemission measurements $[2,3]$, scanning tunneling microscopy, and magnetotransport experiments [4-6]. In particular, spin-momentum locking in TSSs leads to the conversion of a charge current into a spin current, a phenomenon that is commonly addressed as the Rashba-Edelstein effect (REE), while the reverse process is referred to as the inverse Rashba-Edelstein effect (IREE) [7]. In these systems, the leading parameters are the spin-charge interconversion efficiencies: $q_{\mathrm{REE}}=j_{\mathrm{s}}^{3 \mathrm{D}} / j_{\mathrm{c}}^{2 \mathrm{D}}$ for the REE and $\lambda_{\text {IREE }}=j_{\mathrm{c}}^{2 \mathrm{D}} / j_{\mathrm{s}}^{3 \mathrm{D}}$ for the IREE. However, an experimental estimation based on spin pumping-ferromagnetic resonance (FMR) or spin torque-FMR [8-10] is questionable, since TIs are known to chemically react when they are in contact with a ferromagnetic film $[11,12]$. Therefore, a nonlocal architecture where the source of the spin current and the TI are well separated would represent a reliable route to avoid the aforementioned issue.

In this work, we use germanium as a platform for such nonlocal spin-to-charge conversion (SCC) measurements. The spin population is generated by optical spin orientation in $\mathrm{Ge}$, and diffuses as a spin current toward the $\mathrm{Bi}_{2} \mathrm{Se}_{3}$, which

*These two authors equally contributed to the present work. acts as the spin detector. In this way, we totally avoid any ferromagnetic material to generate the spin current.

We probe $\mathrm{SCC}$ at the $\mathrm{Bi}_{2} \mathrm{Se}_{3} / \mathrm{Ge}$ interface kept at room temperature and compare the experimental results with those obtained from a $\mathrm{Pt} / \mathrm{Ge}$ junction in the same experimental conditions. The measurements at the $\mathrm{Pt} / \mathrm{Ge}$ junction allow us to test and validate our microscopic models of spin injection, transport, and nonlocal detection using $\mathrm{Ge}(111)$ as a platform. Indeed, platinum is a prototypical material for spin-to-charge conversion by the inverse spin Hall effect (ISHE) with the well-known spin Hall angle. We first estimate the SCC efficiency at the $\mathrm{Bi}_{2} \mathrm{Se}_{3} / \mathrm{Ge}$ interface, which is found to be of the same order of magnitude as the one of Pt. We then develop microscopic models for spin injection, transport, and nonlocal detection to evaluate the conversion efficiency of the IREE in the junction and find $\lambda_{\text {IREE }} \approx-26 \mathrm{pm}$. Notably, the sign of the SCC is opposite to the one of Pt. To understand this sign reversal, we employ first-principles calculations and demonstrate the existence of Rashba states at the $\mathrm{Bi}_{2} \mathrm{Se}_{3} / \mathrm{Ge}$ interface as a result of strong interfacial hybridization. We find that these states exhibit an opposite spin chirality compared to the one of TSSs in bulk-terminated $\mathrm{Bi}_{2} \mathrm{Se}_{3}$.

\section{SAMPLE PREPARATION}

As a substrate, we use a $2-\mu$ m-thick $n$-doped $\mathrm{Ge}(111)$ layer (doping concentration $N_{\mathrm{d}}=9 \times 10^{16} \mathrm{~cm}^{-3}$ ) epitaxially grown on semi-insulating $\mathrm{Si}$.

The 10-nm-thick $\mathrm{Bi}_{2} \mathrm{Se}_{3}$ film is grown on the $\mathrm{Ge}(111)$ layer by molecular beam epitaxy in the van der Waals regime [13]. (111)-oriented germanium exhibits the proper sixfold symmetry and lattice constant to grow high-quality 


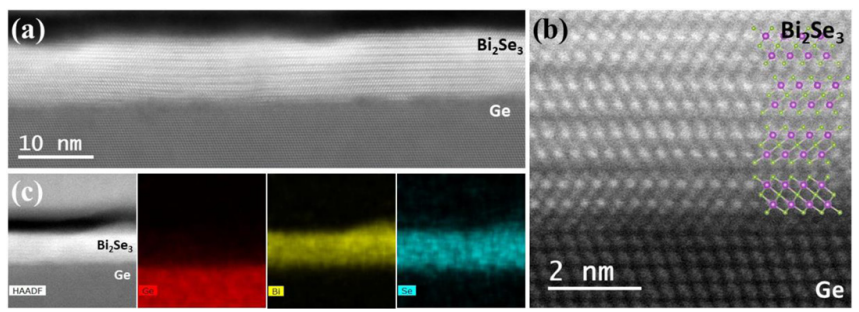

FIG. 1. (a) Low and (b) high magnification transmission electron microscopy cross-section images of the $\mathrm{Bi}_{2} \mathrm{Se}_{3} / \mathrm{Ge}$ interface showing the atomic sharpness and epitaxial relationship. (c) Energydispersive $\mathrm{x}$-ray spectroscopy of the $\mathrm{Bi}_{2} \mathrm{Se}_{3} / \mathrm{Ge}$ stack. From the highangle annular dark-field (HAADF) image, we extracted the elemental maps of $\mathrm{Ge}, \mathrm{Bi}$, and $\mathrm{Se}$.

epitaxial $\mathrm{Bi}_{2} \mathrm{Se}_{3}$ thin films. During the growth, the surface quality and structure are monitored by reflection high-energy electron diffraction (RHEED). Before depositing $\mathrm{Bi}_{2} \mathrm{Se}_{3}$, the $\mathrm{Ge}(111)$ surface was annealed up to $850^{\circ} \mathrm{C}$ in ultrahigh vacuum (UHV) conditions ( $p \approx 5 \times 10^{-10} \mathrm{mbar}$ ) to remove the native germanium oxide. Then, we performed soft argon etching followed by annealing to obtain the $\mathrm{Ge}(2 \times 8)$ surface reconstruction. We first deposited one monolayer of $\mathrm{Bi}$ at room temperature and annealed the substrate until the $\mathrm{Bi} / \mathrm{Ge}(111)-(\sqrt{3} \times \sqrt{3}) R 30^{\circ}$ surface reconstruction appeared in the RHEED pattern. This Bi layer prevents the reaction of $\mathrm{Ge}$ with $\mathrm{Se}$. $\mathrm{Bi}_{2} \mathrm{Se}_{3}$ is then grown by codepositing $\mathrm{Bi}$ and $\mathrm{Se}$ at a substrate temperature of $220^{\circ} \mathrm{C}$. Bi and Se are evaporated from an e-beam evaporator and a Knudsen cell, respectively. Bi and Se evaporation rates are adjusted in order to obtain a high Se:Bi ratio of about 15:1 to limit the presence of Se vacancies in the film. Finally, a 2-nm-thick aluminum layer is grown to protect the $\mathrm{Bi}_{2} \mathrm{Se}_{3}$ layer from oxidation in air. As illustrated in Figs. 1(a) and 1(b) by cross-sectional transmission electron microscopy, we obtain a very sharp $\mathrm{Bi}_{2} \mathrm{Se}_{3} / \mathrm{Ge}$ interface with the $\mathrm{Bi}_{2} \mathrm{Se}_{3}(110) \| \mathrm{Ge}(1 \overline{1} 0)$ epitaxial relationship. The energy-dispersive $\mathrm{x}$-ray spectroscopy maps shown in Fig. 1(c) confirm the elemental sharpness of the interface with only little selenium diffusion in the first atomic planes of germanium. The 15-nm-thick Pt spin detector is also grown in UHV by e-beam evaporation following the same procedure to prepare a clean $\mathrm{Ge}(111)$ surface prior to the $\mathrm{Pt}$ deposition. The RHEED pattern exhibits rings showing the polycrystalline character of the Pt film.

After the growth, we process the samples into small devices as shown in Fig. 2(a) to perform nonlocal spin-to-charge conversion measurements. To do so, we first spin-coat a photosensitive resist on the samples and use laser lithography (at $\lambda=380 \mathrm{~nm}$ ) followed by ion beam etching and soft $\mathrm{O}_{2}$ plasma cleaning to define the $75 \times 10 \mu \mathrm{m}^{2} \mathrm{Bi}_{2} \mathrm{Se}_{3}$ and Pt detection bars using. After a realignment procedure, a 15-nm-thick Pt layer is deposited by e-beam evaporation, on which the $20 \times 2 \mu \mathrm{m}^{2}$ stripes which are exploited for optical spin injection $[14,15]$ are obtained by lift-off. The distance between the stripes is $10 \mu \mathrm{m}$. In a third step, a 70-nm-thick insulating $\mathrm{SiO}_{2}$ layer is deposited by ion beam sputtering followed by lift-off to insulate the $\mathrm{Au} / \mathrm{Ti}$ contacts from the $\mathrm{Ge}$ substrate and prevent direct spin absorption by the contacts. Finally, the $\operatorname{Au}(120 \mathrm{~nm}) / \operatorname{Ti}(5 \mathrm{~nm})$ pads are deposited by
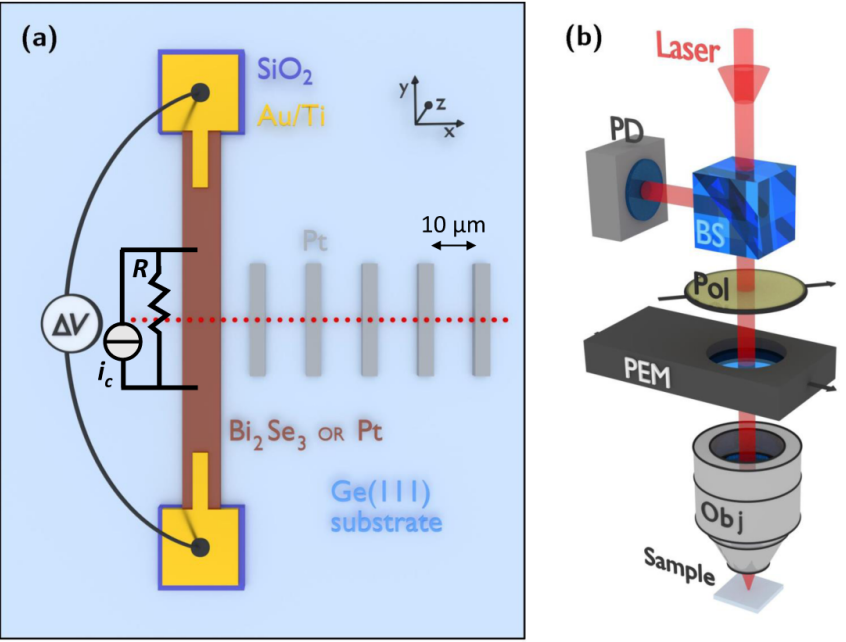

(c)

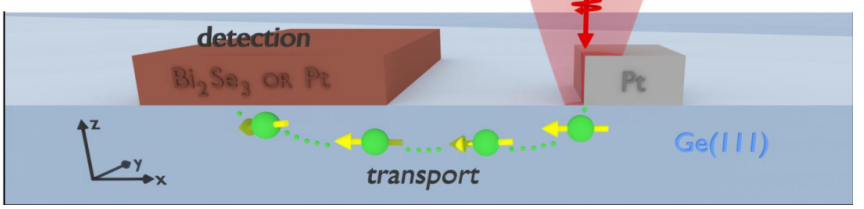

FIG. 2. (a) Sample layout. The vertical brown bar represents the spin detector and is made of either $\mathrm{Bi}_{2} \mathrm{Se}_{3}$ or platinum. The bar is electrically contacted by two Ti/Au pads. $i_{\mathrm{c}}$ is the equivalent charge current generated by the spin-to-charge conversion and $R$ the bar resistance. (b) Confocal microscopy setup. PD, BS, Pol, and PEM represent the photodetector, beam splitter, polarizer, and photoelastic modulator, respectively. (c) Spin generation and diffusion in $\mathrm{Ge}(111)$.

e-beam evaporation and lift-off to contact the $\mathrm{Bi}_{2} \mathrm{Se}_{3}$ or $\mathrm{Pt}$ central detection bar. In this procedure, the interface between the Pt stripes for optical spin orientation and Ge is ill defined due to the lift-off process, with the probable presence of $\mathrm{Ge}$ oxide. This makes those stripes bad spin sinks as compared to the central $\mathrm{Bi}_{2} \mathrm{Se}_{3}$ or Pt detection bars. We can thus neglect spin absorption by these stripes.

\section{METHODS}

The measurements have been performed at room temperature using a confocal microscope, shown in Fig. 2(b). The circularly polarized laser spot is scanning the sample to generate locally a spin accumulation in germanium. In the confocal microscope, the energy of the photons is tuned to the direct $\mathrm{Ge}$ gap $(\hbar \omega=0.8 \mathrm{eV})$ and the circular polarization of the light is modulated by a photoelastic modulator (PEM) at $50 \mathrm{kHz}$. The light is then focused on the sample by an objective with a 0.7 numerical aperture, yielding a laser spot of full-size diameter on the sample of about $3 \mu \mathrm{m}$. The voltage drop $\Delta V$ is then obtained by demodulating with a lock-in amplifier the signal acquired under open-circuit conditions between the $\mathrm{Ti} / \mathrm{Au}$ [see Fig. 2(a)] while the focused light beam raster scans the sample surface.

The working principle of the nonlocal spininjection/detection scheme is illustrated in Fig. 2(c). The 
spin injection is based on the optical spin orientation in Ge [16]. It consists of the absorption of circularly polarized light that generates spin-polarized electron-hole pairs at the $\Gamma$ point of the Brillouin zone. The spin polarization of photogenerated electrons in the conduction band is $P=\left(n_{\uparrow}-n_{\downarrow}\right) /\left(n_{\uparrow}+n_{\downarrow}\right)$, with $n_{\uparrow(\downarrow)}$ being the spin-up (-down) densities referred to the quantization axis given by the direction of the light wave vector in the material. Photogenerated holes are rapidly depolarized due to their very short spin lifetime [17]. If the incident photon energy is tuned to the direct Ge band gap, an electron spin polarization $P=50 \%$ can be achieved [18]. Right after the photogeneration, spin-oriented electrons thermalize from the $\Gamma$ to the L valleys within approximately $300 \mathrm{fs}$, maintaining most of their spin polarization [19]. In the microscope, the laser beam impinges the sample at normal incidence and only an out-of-plane spin polarization is generated by optical spin orientation in Ge, preventing any electrical spin detection by the $\mathrm{Bi}_{2} \mathrm{Se}_{3}$ or Pt bars. The Pt stripes allow us to circumvent this limitation, as already demonstrated in Ref. [14]. In short, when the sample is illuminated with circularly polarized light focused at the edge of a $\mathrm{Pt}$ stripe, the $x$ component $E_{x}$ of the electric field induces charges that generate in the Ge substrate a near-field with a large component in the $z$ direction. The latter is in antiphase with respect to $E_{x}$ because the illumination wavelength of $1550 \mathrm{~nm}$ is significantly shorter than those corresponding to the main plasmonic resonances of the Pt stripe. The combination of the $z$ component of the near-field with the $\pi / 2$ phase-shifted $y$ component $E_{y}$ of the incoming light results in an elliptic field polarization in the $y z$ plane that can generate electrons with spin polarization along the $x$ axis. Opposite spin polarizations are attained at opposite edges of the Pt stripes. To estimate the fraction of photons with in-plane angular momentum when light impinges the edge of a platinum stripe, three-dimensional numerical simulations have been performed by applying finite-difference time-domain simulations $[14,20]$. We reproduce the experimental illumination conditions, with complex dielectric constants $\epsilon_{\mathrm{Ge}}=19+i 0.087$ and $\epsilon_{\mathrm{Pt}}=-21.36+i 74.8$ for $\mathrm{Ge}$ and $\mathrm{Pt}$, respectively [21]. The Stokes parameter $c_{x}=2 \operatorname{Im}\left\{E_{z} E_{y}^{*}\right\}$ is calculated inside the Ge substrate when the focus of the light beam is centered on the edge of a Pt microstructure. In this geometry, the fraction of photons with a projection of the angular momentum along the $x$ axis of the sample is estimated as the ratio between the integral of $c_{x}$ and the integral of the total electric field intensity $I=E_{x}^{2}+E_{y}^{2}+E_{z}^{2}$ over the volume of the Ge film. The result is further normalized to the fraction of impinging photons that are absorbed in Ge, yielding to a final value of $\eta_{\mathrm{g}}=2.2 \%$. The resulting spin accumulations at $\mathrm{Pt}$ stripe edges create spin currents directed to the detection bar with in-plane polarization which are converted into a transverse voltage drop $\Delta V$ caused by the ISHE [22] in the case of detection with Pt, and by the IREE [7] in the case of $\mathrm{Bi}_{2} \mathrm{Se}_{3}$. In both cases, the geometry of spin-to-charge conversion imposes $\Delta V$ to be sensitive only to an electron spin polarization directed along the $x$ axis $[15,23]$. In our analysis, we consider that the spin current generated at $\mathrm{Pt}$ stripes edges in Ge is absorbed at the edge of the detection bar as sketched in Fig. S1 of the Supplemental Material
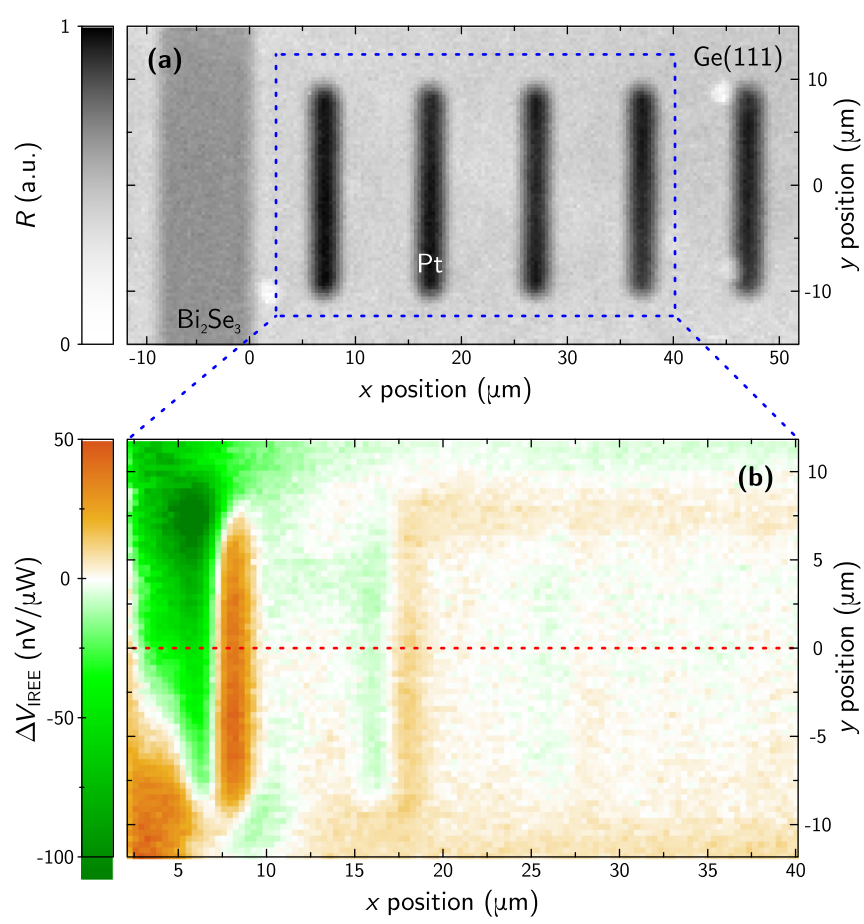

FIG. 3. Reflectivity (a) and IREE map (b) of the $\mathrm{Bi}_{2} \mathrm{Se}_{3} / \mathrm{Ge}(111)$ junction acquired for an incident power $W=18 \mu \mathrm{W}$ at $\hbar \omega=0.8 \mathrm{eV}$. The IREE map is collected over the area identified by the dashed blue rectangle in the upper panel.

[24]: we make the assumption of a point contact absorption [25]. Our detector can be viewed as a generator providing the current $i_{\mathrm{c}}=\gamma i_{\mathrm{s}}$ resulting from the conversion of the spin current $i_{\mathrm{s}}$ into a charge current with an efficiency $\gamma$, in parallel with the resistance $R$ between the $\mathrm{Au} / \mathrm{Ti}$ terminals [see Fig. 2(a)]. The signal we experimentally measure is the spin voltage $\Delta V=i_{\mathrm{c}} R=\gamma i_{\mathrm{s}} R$ (at the modulating frequency of the photoelastic modulator) under open circuit conditions: $\Delta V_{\text {IREE }}$ for $\mathrm{Bi}_{2} \mathrm{Se}_{3}$ and $\Delta V_{\text {ISHE }}$ for Pt. Consequently, the detected spin voltage is proportional to the bar resistance which decreases linearly with the width $w$ of the detector. Here, we have chosen $w=10 \mu \mathrm{m}$ for technical reasons: it is difficult to define top contacts (made of $\mathrm{Au} / \mathrm{Ti}$ ) by laser lithography on bars smaller than $10 \mu \mathrm{m}$. Nevertheless, we detect large spin voltage signals well above the noise level as shown in Sec. IV. Finally, all the microdevice dimensions and characteristic lengths [26] of the system are summarized in Fig. S1 of the Supplemental Material [24].

\section{NONLOCAL SPIN-TO-CHARGE CONVERSION MEASUREMENTS}

We first show the results obtained on the sample with the $\mathrm{Bi}_{2} \mathrm{Se}_{3}$ detector. The reflectivity and the electrical maps are shown in Figs. 3(a) and 3(b), respectively [27]. The electrical map is normalized to the impinging laser power $W$. As expected for a spin-related signal, by illuminating at opposite edges the Pt stripes used for spin injection, the sign of the electric signal is reversed. This can be better visualized in Fig. 4, which shows the profiles, integrated along the $y$ axis, of the reflectivity [panel (a)] and electrical maps [panel (b)]. 

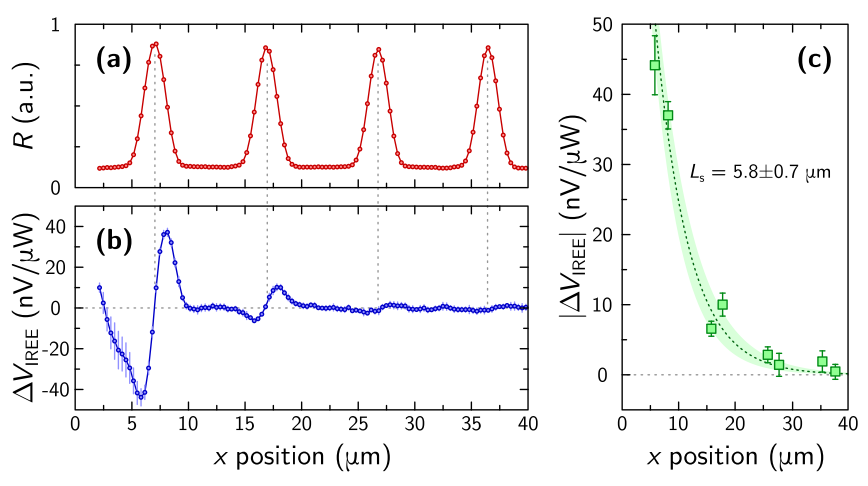

FIG. 4. Reflectivity (a) and IREE (b) profiles signal along the $x$ axis of the sample. (c) $\left|\Delta V_{\text {IREE }}\right|$ as a function of $x . \Delta V_{\text {IREE }}$ is negative for the left edge of each Pt stripe whereas $\Delta V_{\text {IREE }}$ is positive for the right edge. The data are associated by pairs corresponding to each $\mathrm{Pt}$ stripe.

From the latter, we also observe the decrease of the absolute value of the signal when the generation point (i.e., the edge of the illuminated $\mathrm{Pt}$ stripe) moves away from the $\mathrm{Bi}_{2} \mathrm{Se}_{3}$ bar. This signal decay is related to the spin depolarization from the generation to the detection point, which is larger for longer paths [15]. This is illustrated in Fig. 4(c), which reports $\left|\Delta V_{\text {IREE }}\right|$ measured at each $\mathrm{Pt}$ edge as a function of the distance $x$ from the position of the $\mathrm{Bi}_{2} \mathrm{Se}_{3}$ detector.

Although the Pt stripes are a poor spin sink as discussed in the previous section, we can assume that they absorb a small amount of the spins accumulated beneath. We find that the signal dependence as a function of the distance between the injection and the detection points can still be fitted with a single exponential function: $\Delta V_{\text {IREE }} \propto e^{-x / L_{\mathrm{s}}}$ [15], as shown in Fig. 4(c). However, the estimated spin diffusion length $L_{\mathrm{s}}=5.8 \pm 0.7 \mu \mathrm{m}$ corresponds to an effective value shorter than the actual spin diffusion length in bulk Ge, as already discussed in Ref. [15]. This value is indeed slightly shorter than the one reported for $\mathrm{Ge}(001)$ substrates with similar doping [28].

The same analysis, summarized in Fig. 5, has been performed for the sample with the Pt detector. Panels (a) and (b) show the reflectivity and the normalized ISHE map of the sample, respectively, while in panels (c) and (d) we report the profiles along the $x$ axis of the two maps. In panel (e), we plot $\left|\Delta V_{\text {ISHE }}\right|$ at each Pt edge as a function of the distance from the spin detector. We find $L_{\mathrm{s}}=6.0 \pm 1.1 \mu \mathrm{m}$, which perfectly matches the value obtained with the $\mathrm{Bi}_{2} \mathrm{Se}_{3}$ sample. Following the same discussion as for $\mathrm{Bi}_{2} \mathrm{Se}_{3}$, this value slightly underestimates the true spin diffusion length.

Since the spin-injection and transport mechanisms are the same for the two samples, it is possible to quantitatively compare the results obtained with $\mathrm{Bi}_{2} \mathrm{Se}_{3}$ and Pt detectors. First, from Figs. 4(a) and 4(b), we observe that the $\mathrm{Bi}_{2} \mathrm{Se}_{3}$ detection provides a negative (positive) voltage drop when the focused light beam illuminates the left (right) edge of the $\mathrm{Pt}$ injection stripes. Conversely, when SCC is performed via the ISHE in Pt, the signal is positive (negative) at the left (right) edge of the injector microstructures [see Figs. 5(c) and 5(d)]. Hence, the sign of the spin-to-charge conversion in $\mathrm{Bi}_{2} \mathrm{Se}_{3} / \mathrm{Ge}$ is found to be opposite to that in Pt. Previous experiments
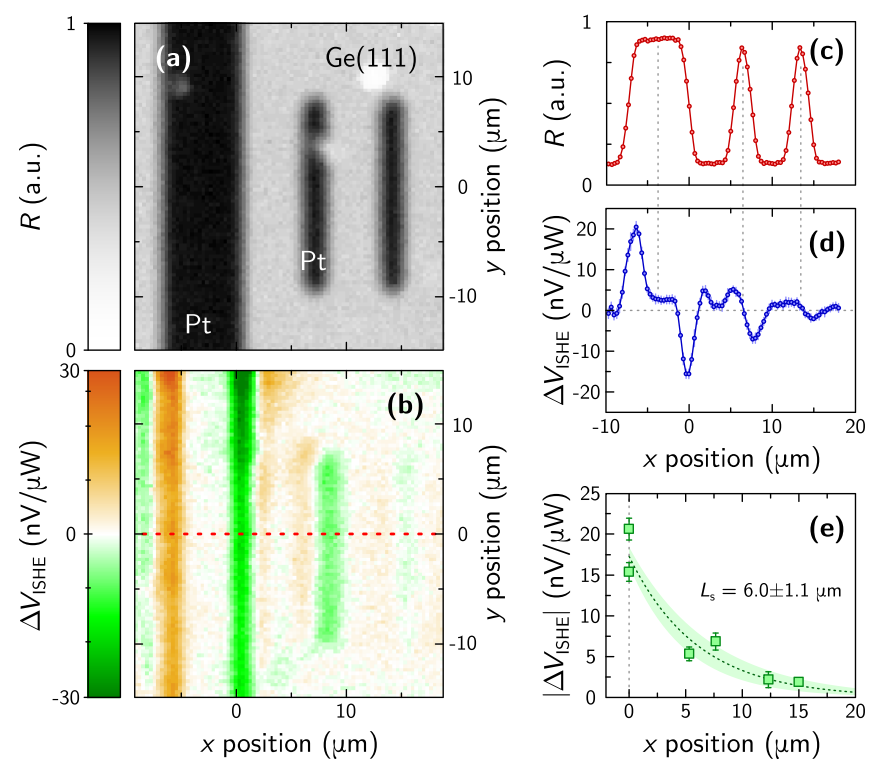

FIG. 5. Reflectivity (a) and ISHE map (b) of the Pt/Ge(111) sample acquired for $W=15 \mu \mathrm{W}$ at $\hbar \omega=0.8 \mathrm{eV}$. Reflectivity (c) and ISHE (d) profiles along the $x$ axis of the sample. (e) $\left|\Delta V_{\text {ISHE }}\right|$ as a function of $x . \Delta V_{\text {ISHE }}$ is positive for the left edge of each Pt stripe whereas $\Delta V_{\text {ISHE }}$ is negative for the right edge. The data are associated by pairs corresponding to each Pt stripe.

were performed to characterize the spin-to-charge conversion in $\mathrm{Bi}_{2} \mathrm{Se}_{3}$ thin films [8-10] and, at variance with our result, the conversion parameter was always measured with the same sign as ISHE in Pt, which we arbitrarily define as "positive." Although the SCC measurements in Refs. [8-10] were carried out with the TI in direct contact with a ferromagnet, this positive sign is also expected from photoemission spectroscopy [2,29] and electrical spin detection [30,31]. Hence, as further discussed in the following, our experimental results suggest that the spin-split states at the $\mathrm{Bi}_{2} \mathrm{Se}_{3} / \mathrm{Ge}(111)$ interface display a substantially different $\mathrm{SCC}$ behavior compared to the ones of a freestanding $\mathrm{Bi}_{2} \mathrm{Se}_{3}$ surface.

Beyond this sign reversal, the comparison of Fig. 4(c) and Fig. 5(e) allows one to estimate the relative spin detection efficiency of $\mathrm{Bi}_{2} \mathrm{Se}_{3} / \mathrm{Ge}$ and $\mathrm{Pt}$. With the light beam focused on the first Pt stripe $\left(x=x_{0} \approx 6 \mu \mathrm{m}\right)$, we measure $\Delta V_{\text {IREE }} / W \approx 40 \mathrm{nV} / \mu \mathrm{W}$ for $\mathrm{Bi}_{2} \mathrm{Se}_{3} / \mathrm{Ge}$ and $\Delta V_{\text {ISHE }} / W \approx 7 \mathrm{nV} / \mu \mathrm{W}$ for Pt. Since the two samples only differ by the spin detector, we conclude that the overall efficiency for spin detection with $\mathrm{Bi}_{2} \mathrm{Se}_{3} / \mathrm{Ge}$ is a factor 5 larger than with Pt. The insulating character of bulk TIs indeed produces higher voltage drops compared to a metal-like Pt for the same charge current.

The macroscopic spin-to-charge conversion parameter is $\gamma=i_{\mathrm{c}} / i_{\mathrm{s}}$, where $i_{\mathrm{s}}$ is the spin current entering the detector and $i_{\mathrm{c}}$ the equivalent charge current across the detection bar, defined as the ratio between the open circuit ISHE or IREE signal $\Delta V$ and the detector resistance $R, i_{\mathrm{c}}=\Delta V / R$. If we assume the same value of $i_{\mathrm{s}}$ for the two samples (due to equal spin injection and transport mechanisms), the relative spin-to-charge conversion efficiency of the materials is $\gamma_{\mathrm{BiSe}} / \gamma_{\mathrm{Pt}}=i_{\mathrm{c}, \mathrm{BiSe}} / i_{\mathrm{c}, \mathrm{Pt}}$. Considering the $\Delta V$ values recorded at $x=x_{0} \approx 6 \mu \mathrm{m}$ and with $R_{\mathrm{BiSe}} \approx 10 \mathrm{k} \Omega$ and $R_{\mathrm{Pt}} \approx 500 \Omega$ 
being the detector resistance, as measured by a four-probe technique, we obtain $\gamma_{\mathrm{Pt}} \approx-3.5 \gamma_{\mathrm{Bise}}$. Here, we stress the fact that $R_{\mathrm{BiSe}}$ and $R_{\mathrm{Pt}}$ account for the possible current shunting in the $\mathrm{Ge}$ channel. However, the electrical current $i_{\mathrm{c}}$ is only sourced in the $\mathrm{Bi}_{2} \mathrm{Se}_{3}$ or Pt bar. The absolute determination of $\gamma$ requires the knowledge of $i_{\mathrm{s}}$. To estimate its value, we start from the spin current excited at the generation time:

$$
i_{\mathrm{s}, 0}=\frac{T W}{\hbar \omega} P \eta_{\mathrm{g}}
$$

where $T W / \hbar \omega$ is the photon absorption rate $(T \approx 0.6$ is the transmittance of $\mathrm{Ge}$ at $\hbar \omega=0.8 \mathrm{eV}$ and $W$ the impinging light power), $\eta_{\mathrm{g}}=2.2 \%$ (see Sec. III), and $P=50 \%$ [16] is the ratio between spin-polarized photogenerated electrons and absorbed photons. The spin current reaching the position of the detector is $i_{\mathrm{s}, 0} e^{-x_{0} / L_{\mathrm{s}}}$, the exponential term accounting for the spin depolarization along the distance $x_{0}$ from the generation point to the detector.

Because of the built-in electric field in the depleted region at the $\mathrm{Bi}_{2} \mathrm{Se}_{3} / \mathrm{Ge}$ and $\mathrm{Pt} / \mathrm{Ge}$ junctions, only a fraction $\eta_{\mathrm{t}}$ of the spin-polarized electrons reaching the position of the $\mathrm{Bi}_{2} \mathrm{Se}_{3}$ bar enters the detector and thus contributes to the measured signal. As shown in Fig. S1 of the Supplemental Material [24], the thickness of the depleted region is on the order of $0.1 \mu \mathrm{m}$ at equilibrium. At this stage, we consider the $\mathrm{Bi}_{2} \mathrm{Se}_{3}$ and Pt detectors as identical spin sinks because of the similar height of their respective Schottky barriers (see below), which also prevent spin backflow in Ge. We calculate $\eta_{\mathrm{t}}$ by exploiting numerical simulations, detailed in Refs. [32,33], which rely on coupled charge and spin drift-diffusion equations. Such simulations take into account the electrostatic effects due to the Schottky barrier, the photovoltaic effect, and the internal Dember field. In particular, the Schottky barrier reduction $\Phi_{\mathrm{ph}}$ produced by the photovoltaic effect at the interface between Ge and the detector is found to be $0.29 \mathrm{eV}$. As a result, we clearly observe that the optically induced spin density gradient between the semiconductor and the detector allows for the injection of spins across the Schottky barrier by means of thermionic emission. In the numerical model, we explicitly account for the height of the Schottky barrier measured by magnetotransport measurements: $\Phi_{\text {bar }} \approx 0.66 \mathrm{eV}$. We find the same value for both $\mathrm{Bi}_{2} \mathrm{Se}_{3} / \mathrm{Ge}$ and $\mathrm{Pt} / \mathrm{Ge}$ junctions as expected from the Fermi level pinning at Ge surfaces [34]. We eventually obtain $\eta_{\mathrm{t}, \mathrm{BiSe}}=\eta_{\mathrm{t}, \mathrm{Pt}}=13 \%$. Since the spin injectors (Pt stripes), the substrate where spins diffuse, the height of the Schottky barrier, and the geometrical characteristics are rigorously the same for both the $\mathrm{Bi}_{2} \mathrm{Se}_{3} / \mathrm{Ge}$ and the $\mathrm{Pt} / \mathrm{Ge}$ heterostructures, the electrical spin-to-charge conversion signal as well as the sign measured on the two samples can be directly compared. We validate the numerical model with $\mathrm{Pt}$, for which the spin Hall angle has been addressed by several works in the literature. In this case, we have measured $\Delta V_{\text {ISHE }} / W=7 \mathrm{nV} / \mu \mathrm{W}$ (obtained for an incident optical power $W=15 \mu \mathrm{W}$ ) at $x=x_{0} \approx 6 \mu \mathrm{m}$, corresponding to $i_{\mathrm{c}}=\Delta V_{\text {ISHE }} / R=210 \mathrm{pA}$. Our numerical estimation of $i_{\mathrm{s}}$ yields $i_{\mathrm{s}}=6 \mathrm{nA}$, giving $\gamma_{\mathrm{Pt}}=i_{\mathrm{c}} / i_{\mathrm{s}} \approx 3.5 \%$. This value is comparable to previously reported ones for evaporated $\mathrm{Pt}$ films [35,36]; therefore we apply the same model to the sample with a $\mathrm{Bi}_{2} \mathrm{Se}_{3}$ detector. At $x=x_{0} \approx 6 \mu \mathrm{m}$, we obtain
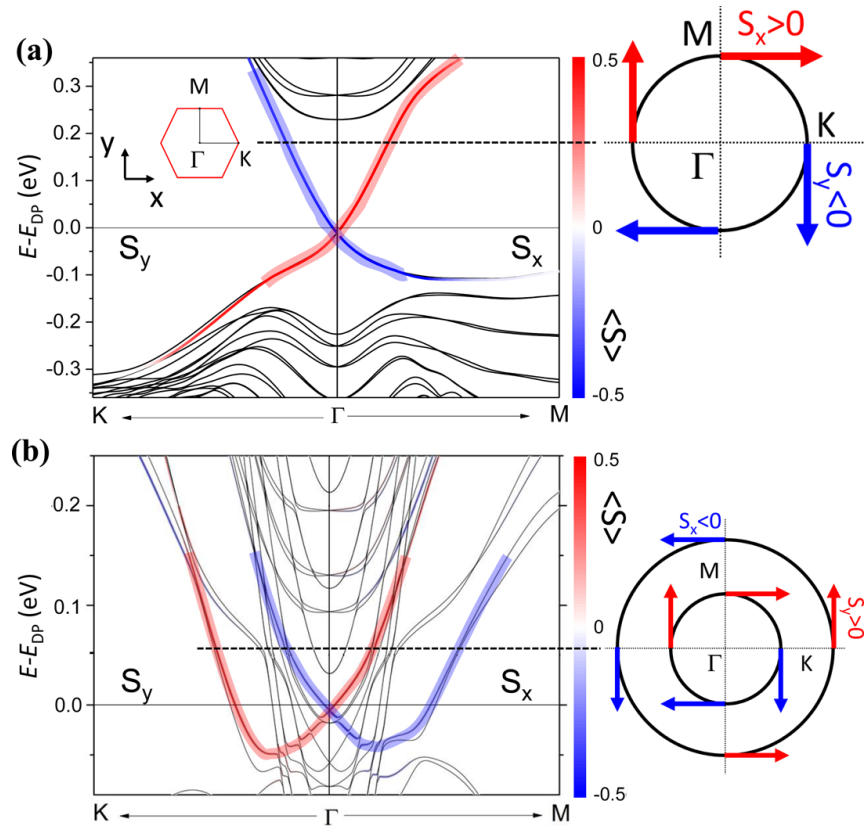

FIG. 6. (a) Electronic band structure with spin-orbit coupling for $8 \mathrm{QL}$ of $\mathrm{Bi}_{2} \mathrm{Se}_{3}$. The red and blue colors indicate the spin texture of the topmost QL projected along the $y$ and $x$ direction for the $\mathrm{K}-\Gamma$ and $\Gamma$-M high symmetry axes, respectively. The corresponding Brillouin zone as well as a specific spin-resolved Fermi contour are reported on the right side. (b) Electronic band structure for the $\mathrm{Bi}_{2} \mathrm{Se}_{3}(8 \mathrm{QL}) / \mathrm{Ge}$ $(3.2 \mathrm{~nm})$ stack. The same color code as in (a) is used for the interface spin texture. A specific spin-resolved Fermi contour is shown on the right side. $E_{\mathrm{DP}}$ corresponds to the energy position of the Dirac point of $\mathrm{Bi}_{2} \mathrm{Se}_{3}$ surface states in (a) and $\mathrm{Bi}_{2} \mathrm{Se}_{3} / \mathrm{Ge}$ interface states in (b).

$\Delta V_{\text {IREE }} / W=-40 \mathrm{nV} / \mu \mathrm{W}$ (measured with $W=18 \mu \mathrm{W}$ ). Hence $i_{\mathrm{c}}=-72 \mathrm{pA}$ and $i_{\mathrm{s}}=7.2 \mathrm{nA}$, yielding $\gamma_{\mathrm{BiSe}} \approx-1 \%$.

Since the spin-to-charge conversion by the IREE occurs in surface or interface states, the relevant parameter describing the SCC efficiency is the inverse Rashba-Edelstein length $\lambda_{\text {IREE }}$, which can be obtained as the product between the macroscopic efficiency parameter $\gamma_{\text {Bise }}$ and the spatial extension $d$ of the surface or interface states in which the conversion takes place. In bulk-terminated $\mathrm{Bi}_{2} \mathrm{Se}_{3}$, the extension of the TSS is $d=3 \mathrm{~nm}$ from Ref. [29]. In our case, the extension of $\mathrm{Bi}_{2} \mathrm{Se}_{3} / \mathrm{Ge}$ interface states is given by first-principles calculations in the next section. Note that to derive $\lambda_{\text {IREE }}$ from $\gamma_{\mathrm{BiSe}}$, we only need to consider SCC occurring in the $\mathrm{Bi}_{2} \mathrm{Se}_{3} / \mathrm{Ge}$ interface states and neglect SCC at the opposite $\mathrm{Bi}_{2} \mathrm{Se}_{3}$ free surface, since the film is thicker than the spin diffusion length [37].

\section{FIRST-PRINCIPLES CALCULATIONS}

In order to understand the opposite SCC signs for the two samples, we have performed first-principles relativistic calculations to unveil the spin-resolved band structure at the $\mathrm{Bi}_{2} \mathrm{Se}_{3} / \mathrm{Ge}$ interface [38-44]. All the computational details are given in the Supplemental Material [24]. We first consider eight quintuple layers (8 QL) of $\mathrm{Bi}_{2} \mathrm{Se}_{3}$. In Fig. 6(a), the band structure is plotted along the $\mathrm{K}-\mathrm{\Gamma}-\mathrm{M}$ direction as shown in the inset. In this particular direction along which $\mathrm{K}-\Gamma$ 
$(\Gamma-\mathrm{M})$ is parallel to the $x(y)$ direction, we plot the band structure weighted by the $y(x)$ spin component $S_{y}\left(S_{x}\right)$ of the topmost QL, as highlighted by the thick red and blue lines. The red (blue) color indicates an in-plane spin pointing in the positive (negative) direction of the axis. We clearly observe the presence of surface states belonging to Dirac cones. Due to spin-momentum locking characteristic of TIs, the in-plane spin helicity of the surface states above the Dirac point (characterized by a positive dispersion) displays a clockwise (CW) chirality, while the helicity of states below the Dirac point (with a negative dispersion) is counterclockwise (CCW). Because of the opposite dispersion relation, both types of chiral states (either above or below the Dirac point) thus lead to a positive $\lambda_{\text {IREE }}$ value and to the same sign of the SCC coefficient as the one observed in platinum [45].

Figure 6(b) displays the band structure of $8 \mathrm{QL}$ of $\mathrm{Bi}_{2} \mathrm{Se}_{3}$ in contact with $3.2 \mathrm{~nm}$ of $\mathrm{Ge}$. Compared with pure $\mathrm{Bi}_{2} \mathrm{Se}_{3}$, many additional electronic states appear due to the strong hybridization with Ge. In Fig. 6(b), we use the same color code as in Fig. 6(a) to highlight the spin texture at the $\mathrm{Bi}_{2} \mathrm{Se}_{3} / \mathrm{Ge}$ interface. Interestingly, due to the strong hybridization between $\mathrm{Bi}_{2} \mathrm{Se}_{3}$ and $\mathrm{Ge}$ orbitals, the bottom Dirac cone is inverted. This cone inversion gives rise to a Rashba-like helical spin texture exhibiting a $\mathrm{CCW}$ chirality of the outer contour for $-0.05 \mathrm{eV}<E-E_{\mathrm{DP}}<0.15 \mathrm{eV}$, where $E_{\mathrm{DP}}$ is the energy of the Dirac point. Therefore, in this energy range, the CCW spin chirality of the outer contour leads to a negative $\lambda_{\mathrm{IREE}}$ value. First-principles calculations thus qualitatively support our experimental observations concerning the sign of the spincharge conversion. We can conclude that SCC does not take place in the TSSs of $\mathrm{Bi}_{2} \mathrm{Se}_{3}$ but rather in hybridized Rashba states at the $\mathrm{Bi}_{2} \mathrm{Se}_{3} / \mathrm{Ge}$ interface. Moreover, from the calculations, we find that those states extend over $2 \mathrm{QL}$ of $\mathrm{Bi}_{2} \mathrm{Se}_{3}$ and 3 monolayers of Ge giving a spatial extension $d=2.6 \mathrm{~nm}$. We then deduce $\lambda_{\text {IREE }}=\gamma_{\text {IREE }} \times d \approx-26 \mathrm{pm}$. It should also be noticed that, by adjusting the position of the Fermi level in Fig. 6(b) with a gate voltage to the $\mathrm{Bi}_{2} \mathrm{Se}_{3} / \mathrm{Ge}$ heterostructure, it could be possible to control both the magnitude and the sign of the spin-to-charge conversion at the interface.

\section{CONCLUSION}

To summarize, we have probed the spin-to-charge conversion at the $\mathrm{Bi}_{2} \mathrm{Se}_{3} / \mathrm{Ge}$ interface by using a nonlocal spin injection/detection scheme. Notably, we measure larger voltage drops with $\mathrm{Bi}_{2} \mathrm{Se}_{3}$ than with the Pt reference, which makes the former an excellent spin detector for future spinbased technologies. We have numerically modeled the spin injection and transport in $\mathrm{Ge}$ to the $\mathrm{Bi}_{2} \mathrm{Se}_{3}$ detector and found an equivalent spin Hall angle close to the one derived for Pt. It corresponds to an inverse Rashba-Edelstein length $\lambda_{\text {IREE }} \approx-26 \mathrm{pm}$. The sign of the spin-to-charge conversion is found to be opposite for $\mathrm{Bi}_{2} \mathrm{Se}_{3} / \mathrm{Ge}$ and Pt. By employing first-principles calculations, we ascribe this behavior to the interfacial hybridization between the topologically protected surface states of $\mathrm{Bi}_{2} \mathrm{Se}_{3}$ and $\mathrm{Ge}$ leading to the formation of Rashba interface states with a spin chirality opposite to the one of states at the free $\mathrm{Bi}_{2} \mathrm{Se}_{3}$ surface. Our results demonstrate that semiconductors constitute a very promising platform for the exploitation of topological insulators in spintronics, where, by gating the heterostructure, spin-to-charge conversion could in principle be tuned in magnitude and sign.

\section{ACKNOWLEDGMENTS}

The authors acknowledge financial support from the ANR project ANR-16-CE24-0017 TOP RISE and from the European Union's Horizon 2020 research and innovation program under Grant Agreement No. 785219 (Graphene Flagship).
[1] M. Z. Hasan and C. L. Kane, Rev. Mod. Phys. 82, 3045 (2010).

[2] D. Hsieh, Y. Xia, D. Qian, L. Wray, J. H. Dil, F. Meier, J. Osterwalder, L. Patthey, J. G. Checkelsky, N. P. Ong, A. V. Fedorov, H. Lin, A. Bansil, D. Grauer, Y. S. Hor, R. J. Cava, and M. Z. Hasan, Nature (London) 460, 1101 (2009).

[3] H. Zhang, C.-X. Liu, X.-L. Qi, X. Dai, Z. Fang, and S.-C. Zhang, Nat. Phys. 5, 438 (2009).

[4] Y. S. Kim, M. Brahlek, N. Bansal, E. Edrey, G. A. Kapilevich, K. Iida, M. Tanimura, Y. Horibe, S.-W. Cheong, and S. Oh, Phys. Rev. B. 84, 073109 (2011).

[5] M. Liu, J. Zhang, C.-Z. Chang, Z. Zhang, X. Feng, K. Li, K. He, L.-L. Wang, X. Chen, X. Dai, Z. Fang, Q.-K. Xue, X. Ma, and Y. Wang, Phys. Rev. Lett. 108, 036805 (2012).

[6] M. Lang, L. He, X. Kou, P. Upadhyaya, Y. Fan, H. Chu, Y. Jiang, J. H. Bardarson, W. Jiang, E. S. Choi, Y. Wang, N.-C. Yeh, J. Moore, and K. L. Wang, Nano. Lett. 13, 48 (2012).

[7] V. M. Edelstein, Solid State Commun. 73, 233 (1990).

[8] M. Jamali, J. S. Lee, J. S. Jeong, F. Mahfouzi, Y. Lv, Z. Zhao, B. K. Nikolic, K. A. Mkhoyan, N. Samarth, and J.-P. Wang, Nano. Lett. 15, 7126 (2015).
[9] S. Shi, A. Wang, Y. Wang, R. Ramaswamy, L. Shen, J. Moon, D. Zhu, J. Yu, S. Oh, Y. Feng, and H. Yang, Phys. Rev. B. 97, 041115(R) (2018).

[10] H. Wang, J. Kally, J. S. Lee, T. Liu, H. Chang, D. R. Hickey, K. A. Mkhoyan, M. Wu, A. Richardella, and N. Samarth, Phys. Rev. Lett. 117, 076601 (2016).

[11] L. A. Walsh, C. M. Smyth, A. T. Barton, Q. Wang, Z. Che, R. Yue, J. Kim, M. J. Kim, R. M. Wallace, and C. L. Hinkle, J. Phys. Chem. C 121, 23551 (2017).

[12] K. Ferfolja, M. Valant, I. Mikulska, S. Gardonio, and M. Fanetti, J. Phys. Chem. C 122, 9980 (2018).

[13] T. Guillet, A. Marty, C. Beigné, C. Vergnaud, M.-T. Dau, P. Noël, J. Frigerio, G. Isella, and M. Jamet, AIP Adv. 8, 115125 (2018).

[14] F. Bottegoni, M. Celebrano, M. Bollani, P. Biagioni, G. Isella, F. Ciccacci, and M. Finazzi, Nat. Mater. 13, 790 (2014).

[15] C. Zucchetti, F. Bottegoni, C. Vergnaud, F. Ciccacci, G. Isella, L. Ghirardini, M. Celebrano, F. Rortais, A. Ferrari, A. Marty, M. Finazzi, and M. Jamet, Phys. Rev. B. 96, 014403 (2017). 
[16] Optical Orientation, edited by F. Meier and B. P. Zakharchenya (North-Holland, Amsterdam, 1984).

[17] F. Rortais, S. Oyarzún, F. Bottegoni, J.-C. Rojas-Sánchez, P. Laczkowski, A. Ferrari, C. Vergnaud, C. Ducruet, C. Beigné, N. Reyren, A. Marty, J.-P. Attané, L. Vila, S. Gambarelli, J. Widiez, F. Ciccacci, H. Jaffrés, J.-M. George, and M. Jamet, J. Phys.: Condens. Matter 28, 165801 (2016).

[18] J. Rioux and J. E. Sipe, Phys. Rev. B 81, 155215 (2010).

[19] F. Pezzoli, F. Bottegoni, D. Trivedi, F. Ciccacci, A. Giorgioni, P. Li, S. Cecchi, E. Grilli, Y. Song, M. Guzzi, H. Dery, and G. Isella, Phys. Rev. Lett. 108, 156603 (2012).

[20] FDTD Solutions 2018b, v. 8.20.1634, Lumerical, Inc., Canada.

[21] E. D. Palik, Handbook of Optical Constants of Solids (Academic Press, 1998).

[22] M. I Dyakonov and V. I. Perel, Phys. Lett. A 35, 459 (1971).

[23] K. Ando, M. Morikawa, T. Trypiniotis, Y. Fujikawa, C. H. W. Barnes, and E. Saitoh, J. Appl. Phys. 107, 113902 (2010).

[24] See Supplemental Material at http://link.aps.org/supplemental/ 10.1103/PhysRevB.101.184406 for (i) a summary of the device dimensions and characteristic lengths of the system, (ii) nonlocal spin-to-charge conversion measurements at the vicinity of the $\mathrm{Bi}_{2} \mathrm{Se}_{3}$ detector and (iii) the details about $a b$ initio calculations.

[25] P. Laczkowski, H. Jaffrès, W. Savero-Torres, J.-C. RojasSánchez, Y. Fu, N. Reyren, C. Deranlot, L. Notin, C. Beigné, J.-P. Attané, L. Vila, J.-M. George, and A. Marty, Phys. Rev. B 92, 214405 (2015).

[26] See http://www.ioffe.ru/SVA/NSM/Semicond/Ge.

[27] The IREE map of Fig. 3(b) does not include the area straddling the $\mathrm{Bi}_{2} \mathrm{Se}_{3}$ detector, since the IREE signal obtained by illuminating the edges of the $\mathrm{Bi}_{2} \mathrm{Se}_{3}$ stripe cannot be directly compared with the one generated at Pt stripe edges, due to the different materials involved in the optical spin injection process. Nevertheless, the corresponding data are shown in the Supplemental Material [24].

[28] C. Zucchetti, M. Bollani, G. Isella, M. Zani, M. Finazzi, and F. Bottegoni, APL Mater. 7, 101122 (2019).
[29] M. Neupane, A. Richardella, J. Sáanchez-Barriga, S. Xu, N. Alidoust, I. Belopolski, C. Liu, G. Bian, D. Zhang, D. Marchenko, A. Varykhalov, O. Rader, M. Leandersson, T. Balasubramanian, T.-R. Chang, H.-T. Jeng, S. Basak, H. Lin, A. Bansil, N. Samarth, and M. Z. Hasan, Nat. Commun. 5, 3841 (2014).

[30] C. H. Li, O. M. J. van't Erve, J. T. Robinson, Y. Liu, L. Li, and B. T. Jonker, Nat. Nanotechnol. 9, 218 (2014).

[31] J. S. Lee, A. Richardella, D. R. Hickey, K. A. Mkhoyan, and N. Samarth, Phys. Rev. B 92, 155312 (2015).

[32] I. Zutic, J. Fabian, and S. Das Sarma, Phys. Rev. B 64, 121201(R) (2001).

[33] G. Isella, F. Bottegoni, A. Ferrari, M. Finazzi, and F. Ciccacci, Appl. Phys. Lett. 106, 232402 (2015).

[34] A. Dimoulas, P. Tsipas, A. Sotiropoulos, and E. K. Evangelou, Appl. Phys. Lett. 89, 252110 (2006).

[35] E. Sagasta, Y. Omori, M. Isasa, M. Gradhand, L. E. Hueso, Y. Niimi, Y. C. Otani, and F. Casanova, Phys. Rev. B 94, 060412(R) (2016).

[36] Y. Huo, F. L. Zeng, C. Zhou, and Y. Z. Wu, AIP Adv. 7, 056024 (2017).

[37] P. Deorani, J. Son, K. Banerjee, N. Koirala, M. Brahlek, S. Oh, and H. Yang, Phys. Rev. B 90, 094403 (2014).

[38] G. Kresse and J. Hafner, Phys. Rev. B 47, 558 (1993).

[39] G. Kresse and J. Furthmüller, Comput. Mater. Sci. 6, 15 (1996).

[40] G. Kresse and J. Furthmüller, Phys. Rev. B 54, 11169 (1996).

[41] P. E. Blöchl, Phys. Rev. B 50, 17953 (1994).

[42] J. P. Perdew, K. Burke, and M. Ernzerhof, Phys. Rev. Lett. 77, 3865 (1996).

[43] U. Herath, P. Tavadze, X. He, E. Bousquet, S. Singh, F. Muñoz, and A. H. Romero, Comput. Phys. Commun. 251, 107080 (2020).

[44] V. Wang, VASPKIT, a postprocessing tool for $a b$ initio code VASP, http://vaspkit.sourceforge.net.

[45] J.-C. Rojas-Sánchez, S. Oyarzún, Y. Fu, A. Marty, C. Vergnaud, S. Gambarelli, L. Vila, M. Jamet, Y. Ohtsubo, A. Taleb-Ibrahimi, P. Le Fèvre, F. Bertran, N. Reyren, J.-M. George, and A. Fert, Phys. Rev. Lett. 116, 096602 (2016). 